\title{
Sacubitril-Valsartan for Heart Failure with Reduced Left Ventricular Ejection Fraction: A Retrospective Study
}

\section{Renato De Vecchis ${ }^{1}$ and Carmelina Ariano ${ }^{1,2}$}

1) Cardiology Unit, Presidio Sanitario Intermedio "Elena d'Aosta", ASL Napoli 1 Centro, via Cagnazzi 29, 80137 Napoli, Italy. e-mail address: devecchis.erre@virgilio.it

2) Division of Geriatrics, Casa di Cura "Sollievo della Sofferenza", viale Cappuccini 2, 71013 San Giovanni Rotondo, Italy. e-mail address: carmelariano@tiscali.it

Corresponding author: Dr Renato De Vecchis, MD Cardiology Unit, Presidio Sanitario Intermedio "Elena d'Aosta", ASL Napoli 1 Centro, via Cagnazzi 29, 80137 Napoli, Italy. E-mail address: devecchis.erre@virgilio.it phone: +390817516932 mobile: +393483313530

\section{Abstract}

Introduction In sacubitril-valsartan (sacub/v), the effects of an angiotensin II receptor blocker (ARB) exerted by valsartan are strengthened by the addition of sacubitril, an inhibitor of neutral endopeptidases. PARADIGM - HF study proved this association to be superior to enalapril in 
reducing both all-cause death and cardiovascular mortality, as well as heart failure (HF) hospitalizations in patients with cardiac insufficiency and reduced left ventricular ejection fraction( HFREF) belonging to NYHA class II-IV. To test whether even in our experience sacub/v is associated with favorable outcomes concerning mortality and morbidity, an outpatient small population of HFREF patients was retrospectively studied, of whom one third was treated with sacub/v instead of conventional therapy with ACE -inhibitors or ARBs.

Methods A retrospective cohort study was carried out to assess the effects of sacub/v in addition to beta-blocker and mineral receptor antagonist (MRA) in a group of HFREF patients in NYHA classes II-III compared with conventional therapy (comprising ACE inhibitor or ARB added to beta-blocker plus a MRA) administered in a second group of HFREF patients with comparable clinical features retrospectively enrolled as controls. In the two groups, the therapeutic regimen was established in accordance with the preferences of the treating physician. Additionally, in both groups, evidence-based drug therapy was supplemented by the adjunct of a loop diuretic, usually furosemide, at variable doses. The primary outcomes of interest were all-cause death and HF hospitalizations. Safety outcomes were symptomatic hypotension, angioedema, hyperkalemia and worsening renal function.

Results Mortality at six months was $6.8 \%$ in patients under therapy with sacub/v versus $34 \%$ in those treated with conventional therapy (odds ratio[OR] $=0.14 ; 95 \% \mathrm{CI}: 0.04-0.49$ ). Moreover, HF hospitalizations in the observation period considered were $4.5 \%$ in sacub/v group versus $59 \%$ in the conventional therapy group $(\mathrm{OR}=0.03 ; 95 \% \mathrm{CI}$ : $0.01-0.14)$. Safety outcomes included in our study (angioedema, hyperkalemia, hypotension and worsening renal function) showed a comparable profile in the two groups, with evidence of good tolerability of sacub/v, except for the side - effect " hypotension" (PAS $<100 \mathrm{~mm} \mathrm{Hg}$ ), found in $15.9 \%$ of patients under sacub/v versus $5.7 \%$ reported in controls $(\mathrm{OR}=3.14$; 95\% CI: $0.94-10.55)$. 
Conclusions In our experience, sacub/v has yielded a strong protection against both all-cause death and HF hospitalizations at six months, in the absence of significant noxious side effects. Nevertheless, considering the retrospective character of the study and the relatively exiguous sample size, further post marketing observational studies would be desirable . In particular, studies aiming at exploring safety of the new pharmacologic principle, namely mainly focusing on hypotension and angioedema, are warranted, in order to validate further this very efficacious molecule for therapy of chronic HF, especially stable HFREF in NYHA classes II-III.

Key words: sacubitril valsartan; heart failure; treatment

\section{MANUSCRIPT}

Background The introduction of sacubitril-valsartan into the officially approved therapeutic repertoire for chronic heart failure (CHF) has been carried out in Italy only on March 12, 2017. Indeed, only since this date, the drug in question, in its various dosage formulations, has received by the competent regulatory authority the official authorization for reimbursement by the Italian National Health Service(1). In the present study we have provided the preliminary data derived from our usage of sacubitril- valsartan, i.e., those concerning the first 8 months of our experience with this drug in the CHF setting. The study was designed as a retrospective cohort study. In fact, it has mainly aimed at investigating some aspects of pharmaco-epidemiology, and has primarily addressed at describing elements and acquisitions derived from the experience achieved by our group. Thus, references to acquisitions from other research groups or other Centres devoted to treatment of heart failure are reported in the text in a quite essential and synthetic manner. Indeed, it's our intention to perform a wider and more detailed examination of the issues related to the use of this drug in a next narrative review. 


\section{Materials and methods}

Patients' selection. The drug was administered, at the discretion of the treating physician, in CHF patients who had various degrees of compromise of hemodynamic and clinical status (Class II or III NYHA). In accordance with the instructions provided by the societal guidelines ( 2 ), NYHA IV patients were excluded from the treatment. Therapy was implemented considering some of the operating models applied by the PARADIGM -HF ( 3 ) study. In our case-record, patients assigned to therapy with sacubitril-valsartan, all of whom had an ascertained diagnosis of CHF dating back at least a year, had to have a history of previous use of inhibitors of the renin-angiotensinaldosterone system( RAAS), maintained for at least three months. In particular, in all of the patients admitted to therapy with sacubitril-valsartan a therapy with ACE- inhibitor or angiotensin-receptor antagonist (ARB) had been already practiced, regardless of clinical outcomes ( clinical improvement, or alternatively, unaltered or worsening outcome). Moreover, the patient had to be free from a possible history of angioedema ( either related to the use of ACE inhibitors or arisen without any identifiable iatrogenic cause). The vast majority of patients who had been admitted to sacubitril-valsartan therapy had already been undergoing a treatment with evidence-based medications,labeled $1 \mathrm{~A}$ according to the recent (January 2016) European Heart Failure Guidelines(4), that is, an association comprising a) a beta-blocker (carvedilol or metoprolol or bisoprolol or nebivolol), b) an ACE inhibitor or ARB and c) a mineralcorticoid receptor antagonist (MRA) selected from the following: spironolactone, eplerenone, canrenone or potassium canrenoate. This association was supplemented by the regular use of a loop diuretic (furosemide or torsemide), given at variable time intervals according to specific patient's clinical picture.

However, the pre-existence of a comprehensive and multi-target, evidence-based therapy for CHF treatment was not considered a binding requirement for the implementation of sacubitril- 
valsartan therapy. Indeed, inclusion criteria were: a condition of NYHA class II-III; a history of prior ACE- inhibitor or ARB therapy with enalapril dose no less than $10 \mathrm{mg}$ daily or equivalent dosage of another ACE- inhibitor or ARB; the absence of previous episodes (one or more) of angioedema; the absence of hypotension, defined by a systolic blood pressure level of $\leq 100 \mathrm{mmHg}$; the absence of severe chronic renal failure ( defined by an estimated glomerular filtration rate [ GFR ],calculated by the Modified Diet in Renal Diseases[MDRD](5)method, no less than $30 \mathrm{ml} / \mathrm{min} / 1.73$ $\mathrm{m} 2$ ), or severe hepatic insufficiency, malignant neoplasia or anemia. Unlike the Paradigm-HF study, the fact of overcoming a well-defined threshold-value of NT-proBNP (e.g., $600 \mathrm{pg} / \mathrm{ml}$ ) was deemed not necessary in order to regard the patient eligible for treatment with sacubitrilvalsartan. Under these conditions, patients with hemodynamic or clinical destabilization (acute heart failure) or patients who had never been treated with ACE-i or ARBs were not admitted to therapy with sacubitril-valsartan. Moreover, during the aforementioned innovative regimen, administration of ACE-i or ARBs or aliskiren was deemed contra-indicated (risk of effect summation). Conversely, in patients treated with sacubitril valsartan, MRA administration was not forbidden. Likewise, beta-blockers were kept except for the possible cases of new, super-imposed complications judged to be contraindications to persistence of pharmacologic beta-blockade. . In addition, depending on the view of the treating physician, a switch from one treatment to another was allowed, but in this case, according to the intention-to-treat principle, the final statistical calculations were performed considering the patient as belonging to treatment group originally assigned.

\section{Modalities adopted for administration of the evidence-based drugs as well as for sacubitril-valsartan}

Target doses to be reached within a reasonably short period of time in the conventional therapy group were to be the following: for beta-blockers, a target dose was established depending on the 
employed molecule (for carvedilol, $25 \mathrm{mg}$ twice daily, for bisoprolol $5 \mathrm{mg}$ twice daily and for nebivolol $5 \mathrm{mg}$ once a day). Likewise, for ACE-inhibitors, the daily target dose was $20 \mathrm{mg}$ for enalapril, $10 \mathrm{mg}$ for ramipril and $20 \mathrm{mg}$ for lisinopril. As an alternative to ACE inhibitors, the use of ARBs was provided for, with a daily target dose of $225 \mathrm{mg}$ for irbesartan, $100 \mathrm{mg}$ for losartan and $160 \mathrm{mg}$ for valsartan. In addition, for MRAs, a daily target dose of $75 \mathrm{mg}$ for spironolactone and 50 $\mathrm{mg}$ for both canrenone and eplerenone was established.

Up-titration of sacubitril-valsartan was carried out provided that in any candidate patient the following measures were accomplished: regular measurements(twice weekly) of systolic blood pressure and periodic determinations( every two months) of serum electrolytes (in particular serum potassium and sodium), serum creatinine and related GFR, whose calculation being obtained using MDRD equation, as well as periodic determinations(every two months) of transaminases, serum NT- proBNP and $\mathrm{Hb}$. The usual criterion was to start assigning to patient 24 + $26 \mathrm{mg}$ of sacubitril-valsartan twice daily ; subsequently, he had to be transferred to dosage of 49 $+51 \mathrm{mg}$ of sacubitril- valsartan twice daily on day 14th, except for cases of obvious intolerance (e.g., symptomatic hypotension, hyperkalemia,etc). Then, after a further 14 days, only in patients with good hemodynamic adaptation and clinical tolerance, the recommended target dose of $97+$ $103 \mathrm{mg}$ of sacubitril -valsartan was provided for, to be assumed once again twice daily.

\section{Study design}

A retrospective cohort study was undertaken by the authors, all of whom were cardiologists with expertise in pharmacoepidemiology who were not directly involved in the therapeutic management of patients undergoing sacubitril-valsartan. Nevertheless, according to the dictates of hospital directorate, they were allowed to have full access to clinical, echocardiographic and hematochemical data of the investigated patients. In collecting data of patients treated with sacubitril- valsartan and those instead undergoing conventional therapy, attention was paid to 
protect patients' privacy as well as to ensure scrupulous preservation of their anonymity. The data from the files of telematic archives were stored on pendrives and later used for the evaluation of the outcomes of interest explored with the aid of statistical programs.

\section{Outcomes of interest.}

The main purpose of the study was the retrospective evaluation of the impact of therapy

with sacubitril- valsartan compared with the conventional regimen on the primary endpoints a) all- cause death and b) heart failure hospitalization. Secondary outcomes were the need to increase by at least $25 \%$ the weekly dose of loop diuretic and impairment by no more than 4 percentage points of the left ventricular ejection fraction. Safety outcomes were: symptomatic hypotension, angioedema, hyperkalemia (serum $\mathrm{K}+>5.5 \mathrm{mEq} / \mathrm{L}$ ) and worsening renal function

( increase in serum creatinine $>0.3 \mathrm{mg} / \mathrm{dl}$ ).

\section{Statistical analysis}

Anthropometric, clinical, anamnestic, haematochemical and echocardiographic data in the 2 groups, the one treated with sacubitril-valsartan and the other subjected to conventional therapy were compared using unpaired samples T test in order to compare the continuous variables. Chi square test with Yates equation was used instead for comparation of dichotomous variables; when needed, Fisher's exact test was adopted for dichotomous variables.

For some binary outcome variables (death at 6 months, heart failure hospitalization, need for diuretic dose increase, LVEF deterioration at 6 months, symptomatic hypotension) comparison between patients exposed and not exposed to sacubitril valsartan was conducted by employing the $2 \times 2$ contingency tables with calculation of the rough ( unadjusted) odds ratio and its $95 \%$ confidence interval.

Cox proportional hazards regression analysis was used to calculate the association of some exposure variables, including the use of sacubitril- valsartan, with the risks of death, hospitalization, increased diuretic dose and worsening LVEF in the course of therapy. Kaplan 
Meier curves were built to estimate with the log-rank test the possible statistical significance of the difference in mortality and hospitalizations found by comparing patients exposed and those not exposed to sacubitril-valsartan.

\section{Results}

Our experience with sacubitril-valsartan was necessarily short , namely no longer than 6 months, given that the marketing of sacubitril-valsartan in reimbursable form by the Italian National Health Service started only in March 2017. Patients who, according to discretionary view of the attending physician, were assigned to conventional treatment, i.e., combination therapy including betablockers, ACE-inhibitors or ARBs plus MRAs, had a profile of anthropometric, clinical and echocardiographic features marked by very strong similarities (see Table 1 ) with those shown by patients assigned to sacubitril valsartan.

Altogether, 132 patients were included in the retrospective study. The mean duration of chronic heart failure treatment in our retrospective study was $5 \pm 1.14$ months (mean \pm standard deviation). Patients assigned to sacubitril valsartan in addition to beta-blocker and MRA were 44, of whom 22 female, whereas the group assigned to conventional therapy with ACE-inhibitors or ARBs in addition to beta-blockers and MRAs consisted of 88 patients, of whom 43 were female. The proportions of patients achieving the target dose in each group were evaluated: for betablockers considered on the whole, the target- dose, i.e., the dose regarded as desirable target at the end of an appropriate up-titration period, was reached in $75 \%$ of cases; for ACE-inhibitors the target dose was reached in $90 \%$ of cases, for ARBs as well as for MRAs in $100 \%$ of cases.

Moreover, for sacubitril valsartan, the target dose was achieved in $80 \%$ of cases.

In patients treated with sacubitril/valsartan, a very important reduction in both all-cause mortality and heart failure hospitalizations was found, in comparison with those subjected to traditional 
therapy. In fact, mortality was $6.8 \%$ in patients under therapy with sacubitril/valsartan versus $34 \%$ in those undergoing conventional therapy $(\mathrm{OR}=0.14 ; 95 \% \mathrm{Cl}: 0.04-0.49)($ Table 2 and Fig 1$)$.

Heart failure hospitalizations in the observation period considered were $4.5 \%$ in sacubitril/valsartan group versus $59 \%$ in the conventional therapy group (OR $=0.03 ; 95 \% \mathrm{Cl}: 0.01$ -

( Table 3 and Fig 1). Also the need to increase diuretic dose in the sacubitril/valsartan group was less frequent: $9 \%$ versus 56.8\% (OR: 0.08; 95\% Cl: 0.03-0.23)( Table 4 and Fig 1 ) . Conversely, a deterioration of no more than 4 percentage points of LVEF was very similar in the two groups : 68.2 versus $62.5 \%$ (nonsignificant OR of $1.29 ; 95 \% \mathrm{Cl}: 0.60-2.77$ )( Table 5 and Fig 1 ).

Safety outcomes included in our study (angioedema, hyperkalaemia, hypotension and worsening renal function) showed a comparable profile in the two groups( Fig 2), with evidence of good tolerability of sacubitril/valsartan, except for the side - effect " hypotension" (PAS <100 mm Hg), found in $15.9 \%$ of patients under sacubitril/valsartan versus $5.7 \%$ reported in controls( Table 6 and Fig 2).

With multivariate Cox proportional hazards regression analysis, use of sacubitril/valsartan has been shown to be a protective factor against the death at 6 months (hazard ratio $(H R)=0.132$; 95\% Cl: 0.0396 to $0.4454 ; p=0.0011$ ) (table 7) with a predictive value greater than that has been exhibited by the age or serum NTproBNP, which both have proved to be risk factors for death at 6 months.

In addition, by means of Cox proportional hazards regression analysis, therapy with sacubitril valsartan has been shown to be associated with decreased risk of heart failure hospitalizations $(\mathrm{HR}=0.0677 ; 95 \% \mathrm{Cl}: 0.0161$ to $0.2845, \mathrm{p}=0.003)($ table 8$)$ 


\section{Discussion}

The truly innovative value of sacubitril/valsartan consists in the fact that it is the first commercially available drug in which the activity of angiotensin receptor blocker( ARB) is favorably integrated and complemented by the combination with a drug, namely sacubitril, capable of enhancing the vasodilator, anti-adrenergic and anti-apoptotic properties possessed by the natriuretic peptide system as well as its action antagonizing the inappropriate rises in ventricular filling pressures. So far, in chronic heart failure, therapeutic activity had focused on the blockade of some neurohormonal systems rather than on the enhancement of the hormonal activity of the peptidergic cardiovascular system, i.e., the cardioprotective system primarily centered around the effects of the B-type natriuretic peptide and atrial natriuretic peptide.

More in detail, it is known that in the setting of volume expansion or pressure overload, the resulting wall stress promotes synthesis of pre-proB-type natriuretic peptide (pre-proBNP) in the ventricular myocardium (6). Subsequently, the peptide is cleaved first to proBNP, then to the biologically active B-type natriuretic peptide (BNP) and the inactive fragment, amino-terminal (NT)-proBNP (7). It is also known that the release of BNP induces improved myocardial relaxation and plays an important regulatory role in response to acute increases in ventricular volume by antagonizing the vasoconstriction, sodium retention, and antidiuretic effects of the activated renin-angiotensin-aldosterone system (RAAS) [8].

Nevertheless, it was found that the favorable vasodilatory and natriuretic properties of natriuretic peptides(NPs) are not sufficient to counteract the noxious effect of RAAS hyperactivation in a significant percentage of cases (8). Thus, the signaling function regarding hemodynamic imbalance, as exerted by NPs when massively secreted, has been judged more important than their possible role in the effective counterbalance against the vasoconstrictive and sodiumretentive systems (8). 
However, some important elements should be emphasized to explain the substantial ineffectiveness of the natriuretic peptide system as a counterweight to the activation of the adrenergic system and RAAS. In particular, the destroying and rapid molecular degradation of BNP, which is carried out by a type of neutral endopeptidase known as neprilysin, makes the functions exercised by BNP rather ephemeral and of overall modest significance. In this regard, it may be useful to underline here that already 15 years ago several attempts were made using inhibitors of neutral endopeptydases, in particular omapatrilat $\quad(9)$ in order to prolong the halflife of natriuretic peptides( BNP and ANP) and thus achieve an enhancement of their counterregulating effects, primarily consisting in lowering ventricular filling pressures and promoting diuresis and natriuresis, so as to antagonize sympatho-adrenergic system and RAAS.

There are some analogies in the structure and pharmacodynamic properties of omapatrilat, and sacubitril, the latter being part of the sacubitril-valsartan molecule. Sacubitril is really a prodrug that is activated to sacubitrilat (LBQ657) by de- ethylation via esterases(10). Sacubitrilat inhibits the enzyme neprilysin (10) which is responsible for the degradation of both atrial and Btype natriuretic peptides.

However neprilysin also catalyzes the degradation process of several other peptides, including bradykinin, a flogosis mediator whose massive release can cause vasodilation, angioedema, and airway obstruction. This concomitant blockade of bradykinin degradation has, however, proved to be much more intense and dangerous when it is associated to use of omapatrilat compared to sacubitril-valsartan .

In fact, in the past, omapatrilat, when was being used as investigational drug, caused relatively numerous cases of severe angioedema involving the glottis with obstruction of the first airways (9). Therefore, safety concerns mainly related to the high incidence of iatrogenic angioedema 
concurred to prevent omapatrilat from being validated by the FDA as a therapeutic agent for cardiac failure. Furthermore, omapatrilat was not found to be superior to enalapril in improving the primary end point of death and hospitalization for heart failure (9).

Conversely, for sacubril-valsartan, based on the safety data of PARADIGM HF(3), the incidence of angioedema was $0.5 \%$, as opposed to a $0.2 \%$ incidence observed in patients treated with the enalapril used as comparator. Therefore, the acceptable impact of this collateral effect with the sacubitril has made it possible to remove the important safety concerns, which had previously led the regulatory authorities to deny the entrance of omapatrilat in the market.

As regards the efficacy outcomes, PARADIGM HF(3) has reported superiority of sacubitril valsartan compared with enalapril, since the new drug has reduced the risk of cardiovascular death and HF hospitalization ( composite endpoint) by $21.8 \%$ with respect to $26.5 \%$ found in patients treated with enalapril. Sudden death was reduced by $20 \%$ compared to the enalapril (HR: $0.80 ; p=0.0082$ ). Moreover, death for irreversible progressive heart failure was reduced by $21 \%$ in in the patients treated with sacubitril/valsartan compared to patients treated with enalapril (HR: $0.79 ; p=0.0338$.

In our retrospective study, we were unable to distinguish the cases of exitus depending on the mode (whether sudden or due to progressively worsening failure). Moreover our data , since they have been calculated from a non-randomized observational study, are burdened by the risk of bias, for example, confounding by indication. Based on our data, we can affirm that sacubitril/valsartan has given a strong protection against both the death at six months (OR: 0.14; 95\% $\mathrm{Cl}$ 0.04-0.49) and HF hospitalizations (OR: 0.03; 95\% Cl: 0.01-0.14).The abovementioned logistic regression results( tables $2-3$ and Fig 1 ) are also largely confirmed by the multivariate Cox proportional hazards regression analysis( table 7 and 8) 


\section{Study limitations}

The main limitations of the study are the small sample size and the "case-control" study design. Indeed, a major characteristic of case-control studies is that data on potential risk factors are collected retrospectively and as a result may give rise to bias.

\section{Conclusions}

Based on our data, sacubitril valsartan has conferred a strong protection against both the death at six months and HF hospitalizations, in the absence of significant harmful side effects. Nevertheless, considering the retrospective character of the study and the relatively small sample size, further post-marketing observational studies are warranted . In particular studies exploring safety of the new pharmacologic principle, e.g., studies centered about the risks of angioedema and hypotension, are required, in order to validate further this very efficacious molecule for treatment of CHF, especially stable cardiac decompensation in NYHA class II-III.

\section{References}

1)Khder Y, Shi V , McMurray JJV , Lefkowitz MP . Sacubitril/Valsartan (LCZ696) in Heart Failure Handb Exp Pharmacol. 2017;243:133-165.

2) Yancy CW, Jessup M, Bozkurt B, Butler J, Casey DE Jr, Colvin MM, Drazner MH, Filippatos G, Fonarow GC, Givertz MM, Hollenberg SM, Lindenfeld J, Masoudi FA, McBride PE, Peterson PN, Stevenson LW, Westlake C. 2016 ACC/AHA/HFSA Focused Update on New Pharmacological Therapy for Heart Failure: An Update of the 2013 ACCF/AHA Guideline for the Management of Heart Failure: A Report of the American College of Cardiology/American Heart Association Task Force on Clinical PracticeGuidelines and the Heart Failure Society of America. J Am Coll Cardiol. 2016 Sep 27;68(13):1476-1488. 
3)McMurray JJ, Packer M, Desai AS, Gong J, Lefkowitz MP, Rizkala AR, Rouleau JL, Shi VC, Solomon SD, Swedberg K, Zile MR; PARADIGM-HF Investigators and Committees. Angiotensinneprilysin inhibition versus enalapril in heart failure. N Engl J Med. 2014 Sep 11;371(11):993-1004 4) Ponikowski P, Voors AA, Anker SD, Bueno H, Cleland JG, Coats AJ, Falk V, GonzálezJuanatey JR, Harjola VP, Jankowska EA, Jessup M, Linde C, Nihoyannopoulos P, Parissis JT, Pieske B, Riley JP, Rosano GM, Ruilope LM, Ruschitzka F, Rutten FH, van der Meer P. 2016 ESC Guidelines for the diagnosis and treatment of acute and chronic heart failure: The Task Force for the diagnosis and treatment of acute and chronic heart failure of the European Society of Cardiology (ESC). Developed with the special contribution of the Heart Failure Association (HFA) of the ESC. Eur J Heart Fail. 2016 Aug;18(8):891-975

5)Levey AS, Bosch JP, Lewis JB, Greene T, Rogers N, Roth D. A more accurate method to estimate glomerular filtration rate from serum creatinine: a new prediction equation. Modification of Diet in Renal Disease Study Group. Ann Intern Med. 1999 Mar 16;130(6):461-70

6)Maeda K, Tsutamoto T, Wada A, Hisanaga T, Kinoshita M. Plasma brain natriuretic peptide as a biochemical marker of high left ventricular end-diastolic pressure in patients with symptomatic left ventricular dysfunction. Am Heart J. 1998 May;135(5 Pt 1):825-32.

7) Nakagawa O, Ogawa Y, Itoh H, Suga S, Komatsu Y, Kishimoto I, Nishino K, Yoshimasa T, Nakao K. Rapid transcriptional activation and early mRNA turnover of brain natriuretic peptide in cardiocyte hypertrophy. Evidence for brain natriuretic peptide as an "emergency" cardiac hormone against ventricular overload. J Clin Invest. 1995 Sep;96(3):1280-7.

8) Maisel A, Mueller C, Adams K Jr et al State of the art: using natriuretic peptide levels in clinical practice. Eur J Heart Fail 2008; 10:824-839

9) Packer M, Califf RM, Konstam MA, Krum H, McMurray JJ, Rouleau JL, Swedberg K. Comparison of omapatrilat and enalapril in patients with chronic heart failure: the Omapatrilat Versus Enalapril Randomized Trial of Utility in Reducing Events (OVERTURE). Circulation. 2002 Aug 20;106(8):920-6. 
10) Gu J, Noe A, Chandra P, Al-Fayoumi S, Ligueros-Saylan M, Sarangapani R, Maahs S, Ksander G, Rigel DF, Jeng AY, Lin TH, Zheng W, Dole WP. Pharmacokinetics and pharmacodynamics of LCZ696, a novel dual-acting angiotensin receptor-neprilysin inhibitor (ARNi). J Clin Pharmacol 2010 Apr;50(4):401-14. 


\section{Fig1 Effects of sacubitril-valsartan}

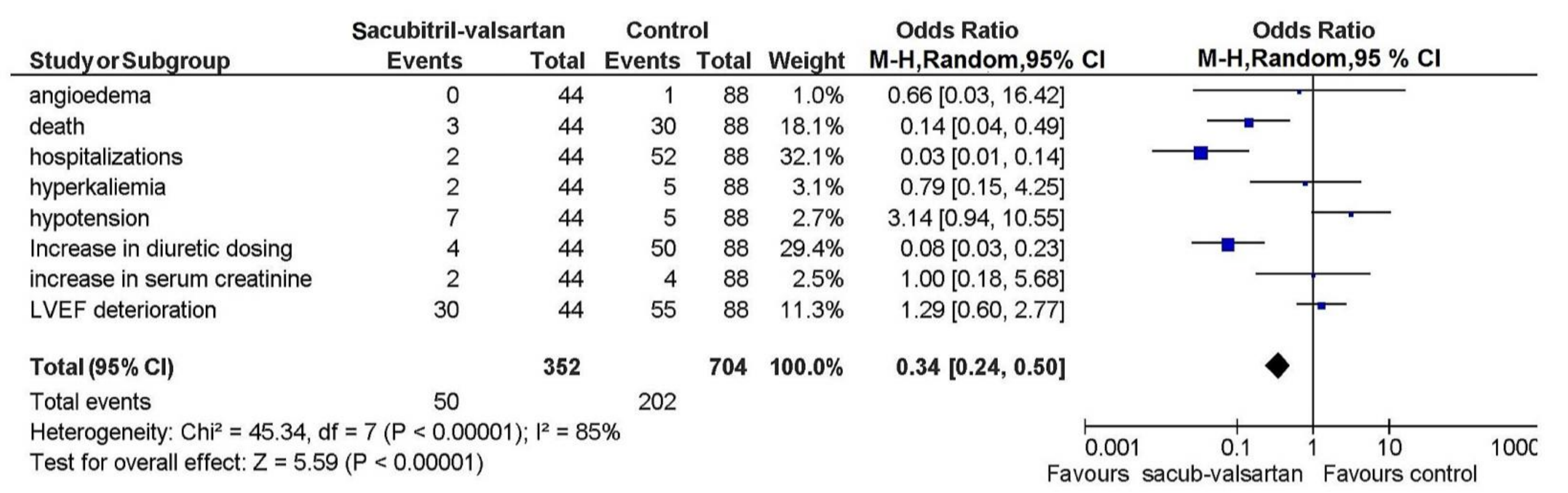




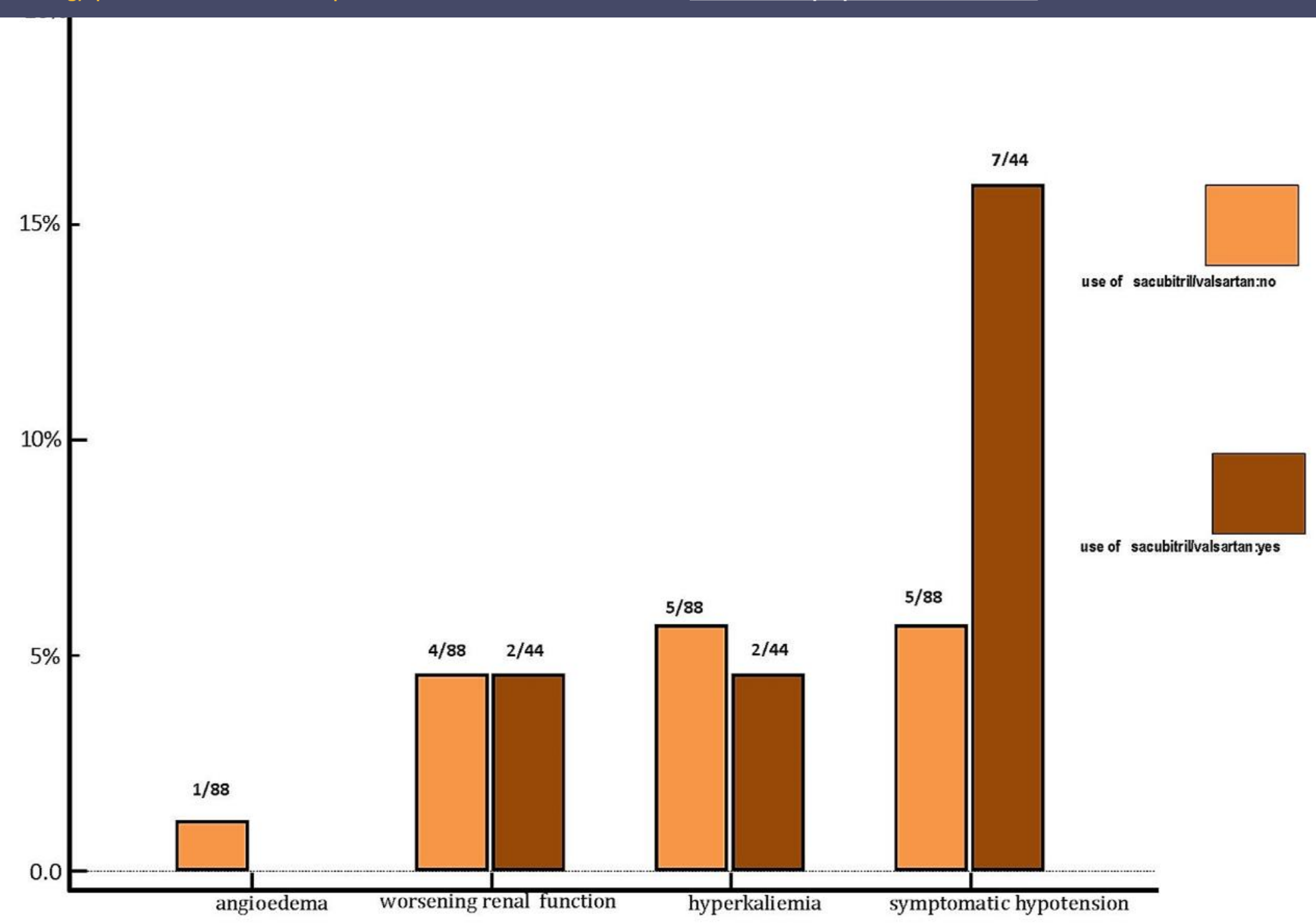

Figure 2 Distribution of side effects (angioedema, worsening renal function, hyperkalemia and symptomatic hypotension) within the population of retrospectively studied CHF patients (no.132) with percentage calculation of cases occurring in subjects treated (No. 44) and in those untreated (no.88) with sacubitril / valsartan.

Legend: $\mathrm{CHF}$, chronic heart failure; worsening renal function: any increase of $\geq 0.3 \mathrm{mg} / \mathrm{dl}$ in serum creatinine compared to baseline 
Comparison of demographics and clinical, laboratory, and echocardiographic features of patients examined in the retrospective study according to whether or not a CHF patient was treated with

\begin{tabular}{|c|c|c|c|}
\hline & $\begin{array}{c}\text { Patients (no. 44) } \\
\text { treated with } \\
\text { sacubitril valsartan }\end{array}$ & $\begin{array}{c}\text { Patients (no. 88) } \\
\text { treated with conventional } \\
\text { therapy, i.e., ACEI or ARBs } \\
\text { (without sacubitril- } \\
\text { valsartan) }\end{array}$ & $p$-Value \\
\hline \multicolumn{4}{|c|}{ Baseline Demographics } \\
\hline Age (years, mean \pm SD) & $76 \pm 5.5$ & $75 \pm 7.5$ & 0.4341 \\
\hline Male sex \% (n) & $70.5 \%(31)$ & $68.2 \%(60)$ & 0,9470 \\
\hline $\begin{array}{l}\text { BMI on admission }\left(\mathrm{Kg} / \mathrm{m}^{2} \text {, mean }\right. \\
\pm \mathrm{SD})\end{array}$ & $28.2 \pm 6.87$ & $27.2 \pm 5$ & 0.3427 \\
\hline $\begin{array}{l}\text { Heart rate at the first visit } \\
\text { (beats/min, mean } \pm \text { SD) }\end{array}$ & $90 \pm 19$ & $85 \pm 20$ & 0.1711 \\
\hline $\begin{array}{l}\text { Heart rate after six months } \\
\text { (beats/min,mean } \pm \text { SD) }\end{array}$ & $64 \pm 18$ & $80 \pm 20$ & $<0.0001$ \\
\hline $\begin{array}{l}\text { SBP at the first visit }(\mathrm{mmHg} \text {, } \\
\text { mean } \pm \mathrm{SD})\end{array}$ & $115 \pm 26$ & $125 \pm 30$ & 0.0617 \\
\hline $\begin{array}{l}\text { SBP after six months }(\mathrm{mmHg}, \\
\text { mean } \pm \mathrm{SD})\end{array}$ & $110 \pm 21$ & $115 \pm 18$ & 0.1574 \\
\hline \multicolumn{4}{|l|}{ Comorbidities } \\
\hline Ischemic etiology of HF \% (n) & $45.4 \%(20)$ & $45.4 \%(40)$ & 0.8529 \\
\hline Valvular etiology of HF \% (n) & $15.9 \%(7)$ & $17 \%(15)$ & 0.9342 \\
\hline CMP-induced HF $\%(n)$ & $27.2 \%(12)$ & $30.6 \%(27)$ & 0.8396 \\
\hline Other cause of HF \% $(n)$ & $11.3 \%(5)$ & $6.8 \%(6)$ & 0,5179 \\
\hline Atrial fibrillation \% (n) & $50 \%(22)$ & $25 \%(22)$ & 0.0074 \\
\hline CABG \% $(n)$ & $22.7 \%(10)$ & $34 \%(25)$ & 0.2550 \\
\hline History of hypertension \% (n) & $56.8 \%(25)$ & $52.2 \%(46)$ & 0.7576 \\
\hline DM on insulin $\%(n)$ & $22.7 \%(10)$ & $28.4 \%(25)$ & 0.6255 \\
\hline COPD \% (n) & $11.3 \%(5)$ & $12.5 \%(11)$ & 1.0000 \\
\hline $\operatorname{ICD} \%(n)$ & $9 \%(4)$ & $10.2 \%(9)$ & 1.0000 \\
\hline $\begin{array}{l}\text { NYHA class IV at baseline } \% \\
(n)\end{array}$ & $2.2 \%(1)$ & $6.8 \%(6)$ & 0.4234 \\
\hline \multicolumn{4}{|c|}{ Hematochemical Variables } \\
\hline $\begin{array}{l}\text { NT-proBNP at the first visit } \\
(\mathrm{pg} / \mathrm{mL}, \text { mean } \pm \text { SD) }\end{array}$ & $800.84 \pm 123$ & $756.22 \pm 129$ & 0.0594 \\
\hline $\begin{array}{l}\text { NT-proBNP after } 6 \text { months } \\
(\mathrm{pg} / \mathrm{mL}, \text { mean } \pm \mathrm{SD})\end{array}$ & $290.5 \pm 90.1$ & $591.47 \pm 213.81$ & $<0.0001$ \\
\hline $\begin{array}{l}\text { Serum creatinine }(\mathrm{mL} / \mathrm{dL} \text {, mean } \\
\pm \mathrm{SD})\end{array}$ & $1.46 \pm 0.55$ & $1.6 \pm 0.4$ & 0.0981 \\
\hline $\begin{array}{l}\text { Serum } \mathrm{Na}^{+} \text {at the first visit } \\
(\mathrm{mEq} / \mathrm{L}, \text { mean } \pm \mathrm{SD})\end{array}$ & $136 \pm 1.55$ & $137 \pm 2.5$ & 0.0166 \\
\hline $\begin{array}{l}\text { Serum } \mathrm{Na}^{+} \text {after } 6 \text { months } \\
(\mathrm{mEq} / \mathrm{L}, \text { mean } \pm \mathrm{SD})\end{array}$ & $138.5 \pm 10$ & $138.4 \pm 8.6$ & 0.9526 \\
\hline $\begin{array}{l}\text { Serum } \mathrm{K}^{+} \text {at the first } \\
\text { visit(meq/L, mean } \pm \mathrm{SD})\end{array}$ & $4.5 \pm 0,6$ & $4.7 \pm 0.9$ & 0.1851 \\
\hline $\begin{array}{l}\text { Serum } K^{+} \text {after } 6 \text { months } \\
(\mathrm{meq} / \mathrm{L}, \text { mean } \pm \mathrm{SD})\end{array}$ & $4.8 \pm 0.65$ & $4.1 \pm 0.85$ & $<0.0001$ \\
\hline \multicolumn{4}{|c|}{ Echocardiographic data at the first visit } \\
\hline LVEF \% (mean $\pm \mathrm{SD})$ & $38.2 \pm 6$ & $37 \pm 5.5$ & 0.2538 \\
\hline
\end{tabular}




\begin{tabular}{|l|c|c|c|}
\hline LVESD $(\mathrm{mm}$, mean $\pm \mathrm{SD})$ & $58 \pm 10$ & $59 \pm 14$ & 0.6733 \\
\hline E/A ratio (mean $\pm \mathrm{SD})$ & $3 \pm 1.25$ & $3.4 \pm 1.35$ & 0.1026 \\
\hline $\begin{array}{l}\text { Deceleration time }(\mathrm{ms}, \text { mean } \pm \\
\text { SD) }\end{array}$ & $136 \pm 22$ & $145 \pm 25$ & $\mathbf{0 . 0 3 6 2}$ \\
\hline
\end{tabular}

Legend SD: standard deviation; BMI: body mass index; SBP: systolic blood pressure; CABG: coronary artery bypass graft; DM: diabetes mellitus; COPD: chronic obstructive pulmonary disease; ICD: implantable cardioverter defibrillator; LVEF: left ventricular ejection fraction; LVESD: left ventricular end-systolic diameter 


\begin{tabular}{|c|c|c|c|c|}
\hline & & \multicolumn{2}{|c|}{ Death over a six-month FU } & \\
\hline \multirow{4}{*}{ 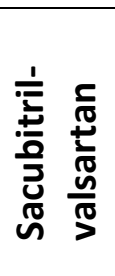 } & & yes & no & \\
\hline & yes & 3 & 41 & 44 \\
\hline & no & 30 & 58 & 88 \\
\hline & TOTAL & 33 & 99 & 132 \\
\hline
\end{tabular}

$\begin{array}{cccc} & \text { Point } & \text { 95\% confidence interval } \\ & \text { estimate } & \text { lower } & \text { upper } \\ \text { Odds ratio (cross product) } & \mathbf{0 . 1 4 1 5} & \mathbf{0 . 0 4 0 4} & \mathbf{0 . 4 9 4 9}\end{array}$

\section{$p($ Fisher exact test $)=0.000516$}

Table 2 The odds of death were significantly lower in patients with chronic heart failure who were taking sacubitril - valsartan ( 44 patients on the whole) in addition to beta-blocker and MRA compared to patients treated with conventional therapy ( 88 patients on the whole), who were taking ACE-inhibitor or ARB in addition to beta-blocker and MRA. Therefore, sacubitril- valsartan has exerted a more favorable effect on mortality compared to conventional therapy with ACE- inhibitor or ARB .

Legend: FU, follow-up; $A C E$, angiotensin-converting enzyme; MRA, mineral-corticoid receptor antagonist; ARB, angiotensin receptor blocker 


\begin{tabular}{|c|c|c|c|c|}
\hline \multirow{2}{*}{} & & \multicolumn{2}{|c|}{ heart failure hospitalizations } & \\
\cline { 2 - 5 } & & yes & no & Total \\
\cline { 2 - 5 } & yes & 2 & 42 & 44 \\
\hline & & 52 & 36 & 88 \\
\hline & Total & 54 & 78 & 132 \\
\hline
\end{tabular}

\begin{tabular}{lccc} 
& Point & \multicolumn{2}{c}{$95 \%$ confidence interval } \\
& estimate & lower & upper \\
Odds ratio (cross product) & $\mathbf{0 . 0 3 3 0}$ & $\mathbf{0 . 0 0 7 5}$ & $\mathbf{0 . 1 4 4 9}$
\end{tabular}

\section{$p$ (Fisher exact test) $<0.000001$}

Table 3 The odds of hospitalization were significantly lower in patients with chronic heart failure who were taking sacubitril - valsartan ( 44 patients on the whole) in addition to beta-blocker and MRA compared to patients treated with conventional therapy ( 88 patients on the whole), who were taking ACE-inhibitor or ARB in addition to beta-blocker and MRA. Therefore, sacubitril-valsartan has prevented hospitalization more efficaciously than conventional therapy with ACE- inhibitor or ARB .

Legend: ACE, angiotensin-converting enzyme; MRA, mineralcorticoid receptor antagonist; $A R B$, angiotensin receptor blocker 


\begin{tabular}{|c|c|c|c|}
\hline & \multicolumn{2}{|c|}{$\begin{array}{c}\text { Increase in loop diuretic dosing } \\
\text { over a six-month FU }\end{array}$} & \\
\hline & yes & no & Total \\
\hline & 4 & 40 & 44 \\
\hline yes & 50 & 38 & 88 \\
\hline Total & 54 & 78 & 132 \\
\hline
\end{tabular}

\begin{tabular}{lcll} 
& Point & \multicolumn{2}{c}{ 95\% confidence interval } \\
& estimate & lower & upper \\
Odds Ratio $($ cross product $)=$ & 0.0760 & 0.0250 & 0.2308 \\
$\boldsymbol{p}($ Fisher exact test $)<\mathbf{0 . 0 0 0 1}$ & & &
\end{tabular}

Table 4 The odds of an increase in doses of $\geq 25 \%$ of loop diuretic ( furosemide or torsemide) with respect to initial dose were significantly lower in patients with chronic heart failure who were taking sacubitril - valsartan ( 44 patients on the whole) in addition to beta-blocker and MRA compared to patients treated with conventional therapy ( 88 patients on the whole), who were taking ACE-inhibitor or ARB in addition to beta-blocker and MRA. Therefore, the use of sacubitril-valsartan has resulted in a less frequent need of diuretic dose augmentation during follow-up compared to conventional therapy with ACE- inhibitor or ARB .

Legend: FU, follow-up; ACE, angiotensin-converting enzyme; MRA, mineral-corticoid receptor antagonist; $A R B$, angiotensin receptor blocker 


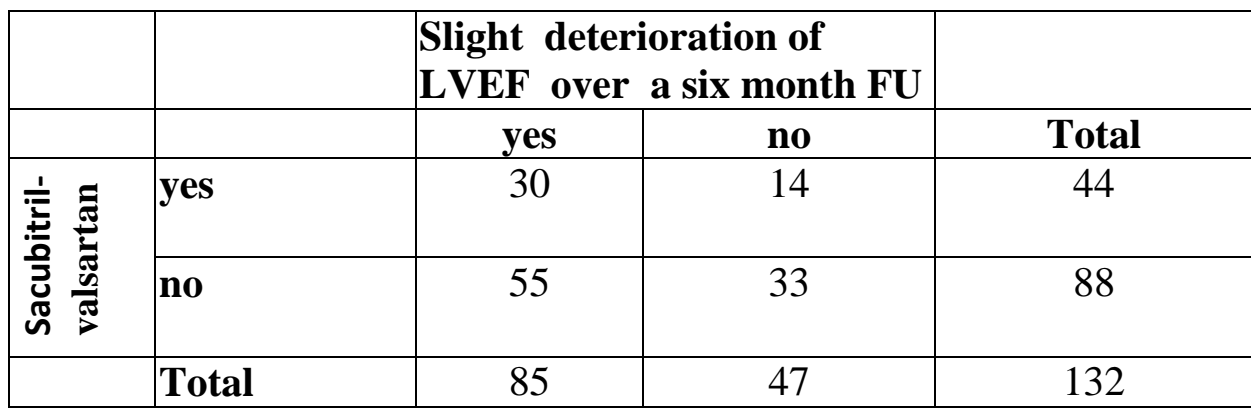

Point $\quad 95 \%$ confidence interval
Odds Ratio (cross product)
estimate
lower
upper
$p($ chi- square corrected according Yates $)=\mathbf{0 . 6 5 2 8 0}$
0.5969
2.7693

Table 5 In patients with chronic heart failure who were taking sacubitril - valsartan ( 44 patients on the whole) in addition to beta-blocker and MRA, the odds of a slight deterioration (no more than $4 \%$ ) of LVEF over a six-month follow-up did not differ from those of patients treated with conventional therapy ( 88 patients on the whole), who were taking ACE-inhibitor or ARB in addition to beta-blocker and MRA. Therefore, the use of sacubitril-valsartan did not yield a significant advantage in protecting left ventricle against progressive enlargement due to underlying heart disease, compared to conventional therapy with ACE- inhibitor or ARB .

Legend: LVEF, left ventricular ejection fraction; FU, follow-up; ACE, angiotensin-converting enzyme; MRA, mineral-corticoid receptor antagonist; $A R B$, angiotensin receptor blocker 


\begin{tabular}{|c|c|c|c|c|}
\hline & & \multicolumn{2}{|c|}{ symptomatic hypotension } & \multirow[b]{2}{*}{ Total } \\
\hline & & yes & no & \\
\hline \multirow{2}{*}{ 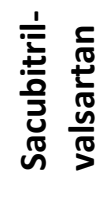 } & yes & 7 & 37 & 44 \\
\hline & no & 5 & 83 & 88 \\
\hline & Total & 12 & 120 & 132 \\
\hline
\end{tabular}

$\begin{array}{ccrr}\text { Point } & \text { 95\% } & \text { confidence interval } \\ \text { estimate } & \text { lower } & \text { upper } \\ \text { Odds ratio }(\text { cross product })= & 3.1405 & 0.9353- & 10.5454\end{array}$

$p($ Fisher exact test $)=0.10361$

Table 6 In patients with chronic heart failure who were taking sacubitril - valsartan in addition to betablocker and MRA, a nonsignificant trend $(p=0.103)$ toward an increased frequency of symptomatic hypotension was detected with respect to patients undergone conventional therapy with ACE-inhibitor(or alternatively ARB), beta-blocker and MRA. Please see also the text.

Legend: ACE, angiotensin-converting enzyme; ARB, angiotensin receptor blocker; MRA, mineral-corticoid receptor antagonist 


\begin{tabular}{|l|l|}
\hline Survival time & Duration of treatment( months) \\
\hline Endpoint & death at 6 months \\
\hline
\end{tabular}

\begin{tabular}{|l|c|c|c|c|c|}
\hline Variable & coefficient & st. error & hazard ratio & $95 \%$ Cl & $p$ \\
\hline Atrial fibrillation & 0.3974 & 0.4485 & 1.4879 & 0.6206 to 3.5677 & 0.3756 \\
\hline age & 0.05737 & 0.01919 & 1.0590 & 1.0201 to 1.0994 & $0.0028^{*}$ \\
\hline sacubitril-valsartan & -2.0185 & 0.6203 & $\mathbf{0 . 1 3 2 9}$ & $\mathbf{0 . 0 3 9 6}$ to 0.4454 & $\mathbf{0 . 0 0 1 1 ^ { * }}$ \\
\hline NT-proBNP & 0.001425 & 0.0005435 & $\mathbf{1 . 0 0 1 4}$ & $\mathbf{1 . 0 0 0 4}$ to 1.0025 & $\mathbf{0 . 0 0 8 7 ^ { * }}$ \\
\hline LVEF at entrance & -0.01904 & 0.02506 & 0.9811 & 0.9344 to 1.0303 & 0.4474 \\
& & & & & \\
\hline
\end{tabular}

Table 7 Outcome variable: death at 6 months. Exposure variables: atrial fibrillation (dichotomous); age (continuous); therapy with sacubitril-valsartan (dichotomous); serum NT-proBNP (continuous); LVEF at entrance (continuous).

Legend: NT-proBNP, N-terminal pro b-type natriuretic peptide; LVEF, left ventricular ejection fraction ${ }^{*}=p$-value which attains statistical significance, i.e., $p<0.05$ 


\begin{tabular}{|l|l|}
\hline Survival time & duration of treatment ( months) \\
\hline Endpoint & heart failure hospitalizations \\
\hline
\end{tabular}

\begin{tabular}{|l|c|c|c|c|c|c|}
\hline Variable & coefficient & Std. error & hazard ratio & $95 \% \mathrm{Cl}$ & $p$ \\
\hline Atrial fibrillation & -0.1910 & 0.4105 & 0.8261 & 0.3710 to 1.8392 & 0.6416 \\
\hline age & 0.01814 & 0.01438 & 1.0183 & 0.9901 to 1.0473 & 0.2072 \\
\hline NT-proBNP & 0.0002895 & 0.0004971 & 1.003 & 0.9993 to 1.0013 & 0.5604 \\
\hline Sacubitril-valsartan & -2.6931 & 0.7364 & $\mathbf{0 . 0 6 7 7}$ & $\mathbf{0 . 0 1 6 1}$ to $\mathbf{0 . 2 8 4 5}$ & $\mathbf{0 . 0 0 3 ^ { * }}$ \\
\hline LVEF at entrance & 0.04061 & 0.02188 & 1.0414 & 0.9980 to 1.0868 & 0.0634 \\
\hline
\end{tabular}

Table 8 Outcome variable: heart failure hospitalizations. Exposure variables: atrial fibrillation (dichotomous); age (continuous); serum NT-proBNP (continuous); therapy with sacubitrilvalsartan (dichotomous); LVEF at entrance (continuous).

Legend: NT-proBNP, N-terminal pro b-type natriuretic peptide; LVEF, left ventricular ejection fraction ${ }^{*}=p$-value which attains statistical significance, i.e., $p<0.05$ 